\title{
Research on Effective Strategies and Teaching Models to Improve High School Physics Learning Interest
}

\author{
Caixia Qi ${ }^{1, a}$
}

\begin{abstract}
${ }^{I}$ School of physical science and technology, Southwest University, Beibei, Chongqing, China a419885986@qq.com
\end{abstract}

\begin{abstract}
Starting from the two subjects of "teacher" and "student", this paper puts forward effective strategies to improve learning interest from multiple angles and all directions. The measures cover teaching materials, classes, homework, rewards and punishments, cultivating habits, overcoming difficulties and so on. Then it summarizes and forms a certain teaching mode from three aspects: before class, in class and after class
\end{abstract}

Keywords: high school physics, learning interest, teaching strategies.

\section{提高高中物理学习兴趣的有效策略和教学模式研究}

\author{
齐采霞 1
}

${ }^{1}$ 西南大学物理科学技术学院, 北碚, 重庆, 中国

a419885986@qq.com

摘要: 本文从 “老师” 和 “学生” 两个主体出发, 多角度、全方位提出提高学习兴趣的有效策略。措施涵盖 教材、课堂、作业、奖惩、培养习惯、攻克疑难等多个方面。然后从教学实施的课前、课堂、课后三方面总结 形成一定的教学模式。

关键词: 高中物理、学习兴趣、教学策略

\section{1. 前言}

物理在社会发展中占据重要地位, 搞好高中物理 教学十分必要。目前我国高中普遍存在 “物理难学, 物理难教” 的现象。高中物理教学中普遍凸显了学科 的难而较少突出学科的鬼力和趣味性, 学生学习物理 普遍缺乏兴趣。新高考最初的 6 选 3 模式下也出现了 弃选物理现象, 虽然有物理较难的原因, 但也说明学 生学习物理兴趣不足。学习兴趣能激发学生的主观能 动性, 所以物理教学更应注重培养学生学习兴趣, 让 学生主动学、乐于学、善于学。培养学生学习兴趣, 搞好高中物理教学, 定能帮助国家选拔和培养一部分 人才, 尤其是在科研和科技方面。

目前在研究学习兴趣方面, 大多数研究都没有综 合考虑影响兴趣的各种因素, 没有考虑教学环节的具 体实施和相互联系, 提出的措施往往是个别的的不系 统的, 例如上课幽默、多做实验等, 但没有具体说明
如何做, 并且这些措施往往只涉及教学环节的某些环 节, 不系统不全面。还有一些措施是理论上的论述, 很难真正实施。

基于以上研究背景和研究现状, 本文从 “老师” 和 “学生” 两个教学主体出发, 多角度、全方位提出 提高学习兴趣的有效策略。说明在提高学习兴趣的各 个教学环节上和各项任务上这两者分别应该怎么做。 措施涵盖教材、课堂、作业、奖惩、培养习惯、攻克 疑难等多个方面。然后从教学实施的课前、课堂、课 后三方面总结形成一定的教学模式。

\section{2. 提高学习兴趣的有效措施}

影响学生学习物理兴趣的因素有很多, 学生自 身、教师教学、课堂设计、教学工具、作业设计等都 会对其产生影响, 而这些因素间又相互影响, 所以在 设计策略时应相辅相成多方面分析。要培养长久的学 习兴趣需要多方措施相互结合并且要坚持较长时间 
慢慢磨合形成, 可以说这是一个复杂又艰辛的实践工 程。本文主要有三点指导思想, 一是强调在 “动” 中 培养兴趣; 二是策略要尽可能考虑到实际教学的各个 环节; 三是提出的策略一定要能够适用于具体教学 中, 要具有实用性。

\section{1. 让学生 “动” 起来, 在实践中提高学习兴 趣}

新课改理念中提到要以学生为主体, 学生是学习 知识的主体, 要提高学生学习兴趣的水平, 就要充分 发挥学生的主观能动性。有时候不是先有兴趣后学 习, 很多时候是先 “动” 起来, 先投入先研究, 然后 在研究和实践过程中培养学习兴趣。

\section{1. 1. 让学生当老师, 每周开展一次物理有趣 小课堂}

物理中有很多有趣的地方, 比如生活中的物理现 象、物理学史或者科学家的小故事、物理小实验、有 趣的物理视频、照片等, 学生们对这些方面也很感兴 趣, 可以让学生自己 “动” 起来, 搜集相关资料, 然 后展示到专门预留给学生的物理趣味课堂中。为了让 高中学生能有足够的时间准备, 可趁假期提前安排同 学准备, 然后保障每次提前一个月左右告知将要开始 讲的同学，让其有足够多的时间思考和准备。可以多 人合作, 形式也可以多样, 但要求有趣味并且可以学 到知识。在进行过程中, 老师可以给学生提供相关的 帮助和指导, 可以帮学生借需要的仪器, 给学生提供 搜集信息的方式等。在笔者的教学实践中, 有的学生 讲述科学家的有关故事, 有的播放或模仿 “加油向未 来” 、“是真的吗? ” 等节目中的精彩实验并解释相 关原理。笔者给学生推荐 “中科院物理所” 微信公众 号, 学生可以关注里面的 “正经玩” 模拟里面的实验, 用身边的器材做小实验小器材, 有些学生还把自制的 电动机、水火箭、无线充电宝等带到物理小课堂上带 领大家一起做。

\section{1.2. 引导学生画创意思维导图}

思维导图对提升学生能力有很大帮助, 但是思维 导图不容易画, 这个方法的初衰是为了用有趣的方法 整理知识, 所以不能让学生觉得很难很枯燥, 因此实 施该方案时可从以下几个方面考虑。(1)让学生有足够 多的时间完成思维导图, 老师提前安排任务, 任务要 小而精。(2)展现形式可以多样化。可以是诗歌、图片、 自编的歌词等, 也可以画上自己喜欢的画, 涂上喜欢 的颜色, 甚至是写一些感悟或者将知识点幽默化, 鼓 励学生用自己喜欢的方式展现, 想怎么写就怎么写。 但是不提倡华而不实, 重点要放在内容上, 形式可以 多样可以美化等。这样可以以长带短, 在实际教学中 学生有用英语来表达知识的、有自己画画的、有贴自 己喜欢的贴纸, 或者是弄一些漂亮的书签之类的, 看 似花花招招, 实则学生会珍惜和 “经营” 这个笔记本。
(3)老师要有耐心, 刚开始有些学生可能积极性不高, 要多鼓励多表扬, 每次挑出一些优秀的案例, 征得学 生同意后展示给同学们观看学习, 让大家觉得很有意 思能有所收获。比如在电场章节, 学生形象反应 “场” 类比武侠小说中 “功力场”画上场源电荷和试探电荷, 配上文字 “来试试我的厉害” ; 还有画简笔画的、把 知识总结成顺口溜的等。很多方法和思想只有学生自 己动手去整理才能有更深的感悟, 并且只有学生真正 学会知识才会从根本上解决学习兴趣的问题。这个过 程千万不要有太过死板的要求, 因为只有慢慢引导让 学生有记录的欲望、整理的欲望才能慢慢带动他们养 成长期的学习兴趣。

\section{1. 3. 让学生合作交流，共同成长}

合作交流主要是在习题课中应用, 或者是针对某 个难点让学生展开讨论, 这种学习方法有助于学生解 决疑难问题, 帮助学生攻克疑难, 还可以提高学生的 学习投入程度。合作交流可以是课堂上的也可以是课 后的, 下面分两点来说明。首先课堂上的合作交流要 想高效且能有条不紊的进行, 需要注意以下几点(1)讨 论前要给学生足够的独立思考的时间。只有学生先独 立思考, 才能把自己的困惑或者想法表达出来, 才能 提高讨论的效果和效率。(2)选择适合学生讨论的问 题。讨论的问题难度要适宜, 最好是学生经过一定思 考后可以解决的问题。(3)讨论目标要明确。讨论的时 候给学生具体说明讨论的内容和讨论的大概时间。(4) 讨论后要有反馈。比如讨论后可以让有结果的小组来 讲解某些题目, 然后老师指点; 或者学生说明讨论后 仍然不理解的地方, 老师带领大家一起分析等。课后 合作交流方面：可以给学生留一些具有探讨性的题 目, 让学生相互合作完成。课后相互交流的题目要尽 可能有意思, 让学生有探究的欲望。比如可以留发散 性作业, 可以把实验仪器给学生让他们分小组做实 验, 或者让他们用生活化的器材做一个实验, 比如可 以让学生课下合作做一个小型电动机、做全反射的实 验等; 也可以是让他们讨论一些有意思的问题的原 理, 或者是争辩一个问题等。实践证明, “合作交流” 能有效帮助学生解决问题。

\section{1. 4. 让学生充分参与课堂}

课堂是教学的主阵营, 学生是课堂的主体, 要提 高学生学习物理的兴趣, 首先要利用好课堂。课堂中 应坚持 “以学生为本, 以学生为主体” 的原则, 充分 发挥学生主观能动性, 让学生多参与课堂, 可以提高 课堂氛围有效减少课堂消殆情况, 可以帮助提高课堂 效率, 从而提升学生学习物理听物理课的兴趣。参与 课堂的方式有很多, 笔者主要采用以下几种(1)小组合 作交流 (这一点在前文中有具体讲解) (2)学生分组实 验。实验是学生比较喜欢的, 可以把握住每次做实验 的机会, 尽可能让学生多参与。(3)让学生多表达自己 的想法。如有些疑难地方, 可以问问学生哪里不理解, 当时是怎么想的, 让学生参与进来。在实践时发现学 
生其实很喜欢听别的同学讲不会的地方或者错误的 原因, 有些还能找到共鸣, 然后老师顺着他们的想法 分析他们不理解的地方和错误的原因, 学生不仅能找 到自己错的地方, 并且印象深刻。但是这个方法需要 老师有一定的带动能力, 刚开始实施这个方法时, 学 生可能不太愿意表达或者说不明白自己的想法, 老师 不要急, 要有耐心慢慢引导。等学生适应这种方法后 学生会发现这个方法能有效解决他们困惑, 一些积极 的学生甚至会主动表达自己想法。通过这种方法, 可 以有效调节课堂氛围, 让学生注意力更集中。(4)可以 鼓励学生当小老师, 自己讲题。可以提前安排学生准 备, 然后上课的时候学生讲解, 老师辅助并点评, 多 鼓励多指导, 学生在这个过程中会更投入物理学习, 也会慢慢克服上课不敢说、说不好的心理障碍, 慢慢 学生的表达能力、逻辑思维能力等都会得到提升, 当 然这样学生也会慢慢喜欢物理课堂。让学生参与课堂 的方式还有很多, 如让学生做游戏, 提问学生回答某 个问题, 安排学生演版, 让学生齐声朗诵, 让学生总 结等。方法有很多, 但笔者更喜欢无拘束的课堂, 最 好是老师和学生能互相交流, 当然不是所有的 “参与” 学生都喜欢, 要结合自己的教学实际, 找到适合自己 也适合自己学生的参与模式。参与的方法可能每个老 师不尽相同, 看法也不尽一致, 但是不可否认让学生 有效的参与课堂, 可以让学生敢于思考、勤于思考、 善于思考, 能让给学生提高课堂效率, 提高学习投入 程度, 这种方法长期实施可以慢慢培养学生学习物理 的兴趣。

总之, 从学生出发来提高学生的学习兴趣, 笔者 认为重点在于一个 “动” 字, 让学生主动学习、主动 参与、主动探究, 比老师把知识 “塞” 给他们, 让他 们被动学习效果要好的多。笔者认为 “动” 或许不能 帮助所有的学生提升物理兴趣, 但 “不动”、“不参 与”、“不思考” 兴趣更无从培养。

\section{2. 老师综合把控, 全方位提升学生学习兴趣}

教师在教学中占据着重要地位, 是知识的传播 者, 是方法的实施者, 是情感的交流者。在提升学生 学习兴趣方面, 老师要做的事情很多, 教学的安排、 课堂的设计、教材的处理、奖惩的实施、情感的建设、 作业的安排等等, 方方面面老师都要做一个思考。关 于教学的每一个环节老师都要考虑在内, 多方面结 合, 才能最大限度的提升学生的学习兴趣, 毕竟学习 兴趣的培养不是依赖于某一个教学环节或者某一种 教学方法, 更不依赖于某一节课或某一个题。应当承 认的是培养学生学习兴趣这件事情是一项工程, 需要 全方位考虑, 统筹把控。结合教学经验笔者觉得提升 学生学习兴趣老师可以采取以下几点措施。1、每节 课提前备好 $3 \sim 5$ 个调节点。这些调节点可以是幽默的 语言、可以是故事、也可以是学生的参与, 或者是结 合着知识点或者某个题的一个形象化类比, 最好能逗 学生乐一乐。不同老师、不同课堂调节点是不一样的, 但是一定要有所准备 “有备而讲”。所谓课堂, 知识
讲的好是必要的, 但是是不够的, 不是知识讲的好讲 的清楚学生就会全神贯注, 更主要的是要讲的让学生 能听进去, 让学生愿意听。2、精备物理知识, 让知 识更丰富更有趣。老师可以采取以下三种措施丰富物 理知识（1）多做实验, 自制物理实验仪器。（2）引 进物理学史, 丰富物理课堂。（3）多将物理与生活 联系, 列举生活实例。3、注重师生情感建设。老师 可以多和学生谈心, 了解学生想法, 帮助学生解决学 习问题。老师也可以制定合理的奖惩措施, 比如学生 成绩好了可以奖励一个小礼品, 或是满足学生一个小 心愿等。4、思考作业的设置, 尽力让学生喜欢做作 业。作业要有针对性不要多而杂, 最好是挑选些与当 天课堂上所讲的知识有密切联系的, 让学生有成就感 和获得感。然后作业设置要有梯度, 先易后难, 可设 置附加题照顾到不同程度学生。周末可设置开放性作 业, 让学生做实验搜资料等。批改作业时可结合奖惩 措施, 奖励表现优异的, 还可以写评语进行情感交流 等。为降低作业枯燥性, 可以安排学生轮流出题, 主 要是出已学习的内容, 同学们相互直接更能理解自己 不会的地方, 并且因为同学关系做题的感觉也会不一 样。5、多角度帮助学生攻克疑难。“疑难” 可以说 是影响学生学习物理兴趣的一个重要因素, 很多学生 就是因为物理难才不愿学物理, 有些甚至产生畏难情 绪对物理学习很抵触, 所以要想提升学生学习兴趣某 种程度上来讲需要想办法帮助学生攻克疑难。攻克疑 难可以采取以下几种措施 (1)要注重方法的总结, 注重 讲练结合。(2)借助实验或仪器演示。(3)小组讨论。(4) 阶梯法, 层层递进。(5)注重学生心理上的疏导。

\section{3. 提升学生学习兴趣的教学模式}

本文策略从 “学生” 和 “教师” 两大方面说明提 高学生学习兴趣的有效策略, 为更好的解读有关策 略, 特从课前、课堂、课后三方面将策略归纳整理, 形成以下模式。

课前方面主要从老师和学生入手, 说明各自的分 工。要想提高学生学习兴趣, 教师需要在备课方面做 大量工作以克服课堂疲备期和低效期。笔者认为好的 课堂绝不是上课的时候才形成的, 而更强调运筹帷幄 之中, 尤其是想要课堂幽默课堂氛围轻松, 更是需要 提前设想好调节点。课前准备教学模式图见图 1 .

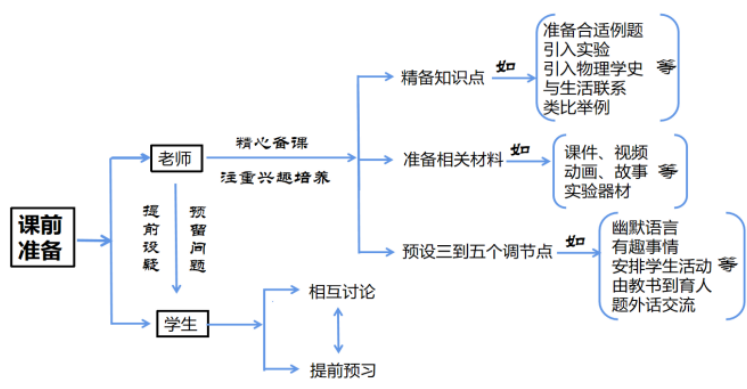

图 1 课前准备模式图

课堂环节模式, 笔者将课堂分为适应期、投入期、 
低效期和疲急期。然后分别说明各个时期老师和学生 的状态和任务。课前准备的调节点主要应用在导入、 低效期和疲急期。这样设计的课堂一方面符合学生心 理状况, 有助于提高课堂效率, 另一方面融合了多种 元素可以有效应对学生的抵抗低效期和疲急期, 有助 于提高学生学习兴趣, 能有效提高课堂氛围。并且这 种模式笔者写清楚了入手点和注意点, 具有可复制性 和推广性。课堂环节方面的模式图见图 2

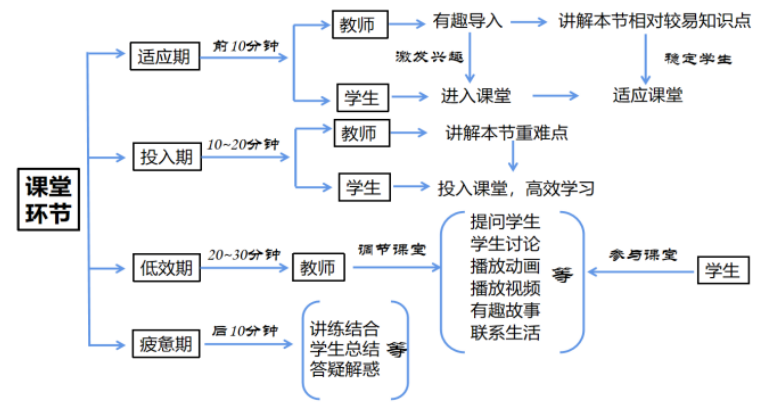

图 2 课堂环节模式图

课后笔者从作业和情感建设两个方面说明如何 提升学生学习兴趣。作业布置方面为避免千篇一律的 资料和卷子, 可以让学生自己出题, 同时可以给学生 留一些操作性的作业, 充分发挥学生主观能动性, 充 分调动学生去发掘物理学科鬼力, 这部分在前面策略 “每周一次物理小课堂中有具体说明” 在这里为了将 策略容纳到一起而将其归为作业设置方面。课后教学 模式见图 3.

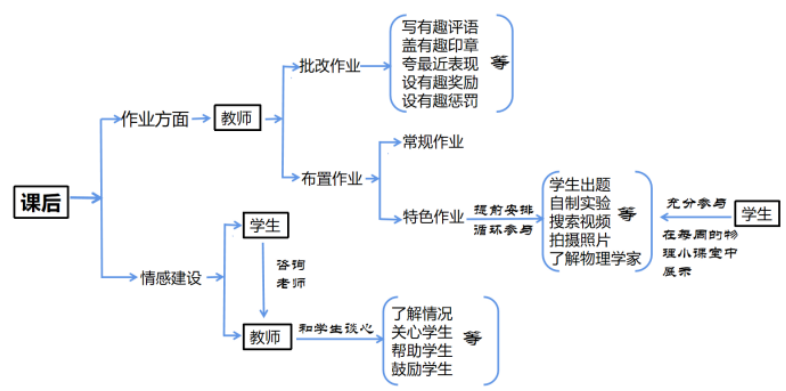

图 3 课后模式图

该部分从课前、课堂、课后三方面将前文中提出 的教师和学生方面的教学策略融合整理起来, 形成一 定备课、授课模式, 具体说明了如何在一线教学中提 升学生学习物理的兴趣。

\section{4. 结论}

本文根据实际教学情况, 结合本人教学经验提出 提高学生高中物理学习兴趣的有效策略。学生方面主 要强调在 “动” 中培养兴趣, 老师方面强调统筹把控, 全方位提高学生学习兴趣。文章从多方面考虑, 各种 对策相辅相成, 并且侧重操作介绍, 全面而又具有实 践意义。

\section{REFERENCES}

[1] Jiang Hui. Investigation and Countermeasures of high school physics learning interest [D]. Fujian Normal University, 2012

[2] Lu Xiaodan, Gao Weiqi. Strengthening the emotional ties between teachers and students and building a harmonious relationship between teachers and students $[\mathrm{J}]$. Science and education collection (last ten days), 2008, (10): 21-24

[3] LV Minfeng. On physics experiment teaching in senior high school [J]. Science weekly, 2011 (02): 182

[4] Meng ronghua. How to cultivate students' interest in learning physics [J]. Reading, writing and calculation: education guide, 2013 (11): 2

[5] Yang Jiangyuan. Research on Cultivation Measures of physics learning interest of senior one students [D]. Henan University, 2013 